\title{
Monochromatic geometric $k$-factors in red-blue sets with white and Steiner points
}

\author{
D. Garijo, M. A. Garrido, C. I. Grima, A. Márquez \\ A. Moreno-González, J. R. Portillo, P. Reyes, \\ R. Robles, and J. Valenzuela ${ }^{1,2}$
}

\author{
Dpt. Matemática Aplicada I \\ Univ. of Seville \\ Seville, Spain
}

\begin{abstract}
We study the existence of monochromatic planar geometric $k$-factors on sets of red and blue points. When it is not possible to find a $k$-factor we make use of auxiliary points: white points, whose position is given as a datum and which color is free; and Steiner points whose position and color is free. We present bounds on the number of white and/or Steiner points necessary and/or sufficient to draw a monochromatic planar geometric $k$-factor.
\end{abstract}

Keywords: $k$-factor, perfect matching, red-blue sets, white points, Steiner points.

1 Partially supported by projects MTM2008-05866-C03-01 and P06-FQM-01649.

2 Email: dgarijo@us.es, vizuete@us.es, grima@us.es, almar@us.es, auxiliadora@us.es, josera@us.es, preyes@us.es, rafarob@us.es, jesusv@us.es 


\section{Introduction}

We consider two disjoint sets $R$ and $B$ of red points and blue points in the plane, respectively, such that no three points of $R \cup B$ lie on the same line. For a survey on discrete geometry on red and blue points in the plane we refer to $[2]$.

Given a set of points $S$ in the plane, we call a geometric $k$-factor of $S$ to a planar k-regular graph embedded in the plane whose vertices are the points of $S$ and whose edges are straight-line segments. A geometric matching of $S$ is a geometric 1-factor. Given two sets $R$ and $B$ of red and blue points, we calla geometric k-factor of $R \cup B$ to be monochromatic it its edges join points with the same color.

From now on, for the sake of simplicity we will assume the $k$-factors to be geometric and monochromatic.

We look for monochromatic planar geometric $k$-factors on sets of red and blue points. In most of the cases it will be necessary to consider auxiliary points: white points, whose position is given as a datum and which color is free; and Steiner points whose position and color is free. We present bounds on the number of white and/or Steiner points necessary and/or sufficient to draw a monochromatic planar geometric $k$-factor.

Through this paper we will make a wide use of the following result [1],

Theorem 1.1 [1] Let $R \cup B$ a set of $n$ red and blue points. Then the following two statements hold:

(i) There exists a monochromatic matching which covers at least $0.8571 n$ points of $R \cup B$. There exists an algorithm for finding such a matching in $O\left(n^{2}\right)$ time.

(ii) There exists a configuration $R \cup B$ for which every monochromatic geometric matching covers at most 0.9871 n points of $R \cup B$.

\section{Monochromatic matchings}

There is an immediate extension of the Theorem 1.1 to monochromatic perfect matchings when the use of Steiner points is allowed,

Corollary 2.1 Let $R \cup B$ a set of $n$ red and blue points. Then $(1-0.8571) n$ Steiner points are always sufficient in order to obtain a monochromatic perfect matchings. Moreover there are sets that require at least $(1-0.9871) n$ Steiner points. 
If we allow only white points, we have the following result,

Theorem 2.2 Let $R \cup B$ a set of $n$ red and blue points. Then $n$ white points are always sufficient and sometimes necessary in order to obtain a monochromatic perfect matching.

Proof (Sketch) First we proof the sufficient condition. It is well-known [3] that if $|R|=|B|$, then there exists a non-crossing bichromatic geometric planar perfect matching on $R \cup B$. So we connect each colored point with a white one and assign it the corresponding color.

A situation where $n$ white points are necessary is shown in Figure 1.

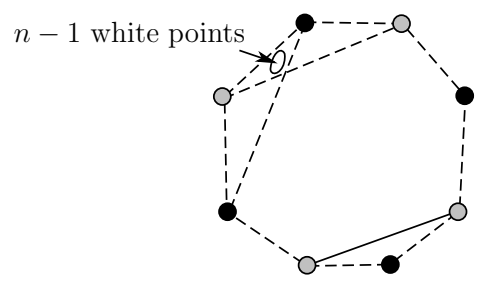

Fig. 1. Any monochromatic segment joining two colored point isolate an odd number of points of the other color.

Finally, if we combine white and Steiner points we obtain the following result,

Theorem 2.3 Given a set of $n$ red and blue points and $k$ a positive integer, then $n-k$ white ones and $\lfloor(1 / 3+0.15) k\rfloor$ Steiner points are always sufficient in order to obtain a monochromatic perfect matching.

Proof (Sketch) Let $S$ be the set of the colored and white points. We look for a pair of consecutive red or blue points on $C H(S)$, or for a white point with a colored neighbor. When we find one of such pairs we match them and remove the points from $S$, update $C H(S)$ and repeat the procedure. If $C H(S)$ is made of alternate red and blue points we make use of Steiner points. Acting in this way we reduce the number of points until either there is the same number of colored points than of white points in $S$ and we apply Theorem 2.2, or all the white points have been removed, and we make use of Corollary 2.1 to obtain a matching. 


\section{Monochromatic k-factors}

We focus now in construct a $k$-factor of $R \cup B$ instead a perfect matching, by making use of Steiner and/or white points. We begin with a result related to 2 -factors and Steiner points,

Theorem 3.1 Let $R \cup B$ a set of $n$ red and blue points. $\lfloor n / 2\rfloor$ Steiner points are always sufficient and sometimes necessary to obtain a 2-factor. This 2factor can be found in $O(n \log n)$ time.

Proof (Sketch) Regarding the necessary condition it suffices to consider the colored points on alternate convex position. Any segment matching two blue points left a red one isolate, requiring at least one Steiner point to join it to the next red point.

To construct a 2-factor we sort the point the points of $R$ and $B$ lexicographically by polar angle and distance from a point inside their convex hulls and construct two cycles $C_{R}$ and $C_{B}$ by making use of Steiner points, drawn as squares in Figure 2

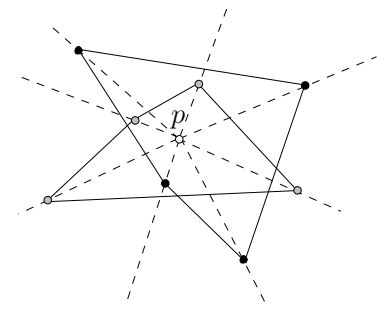

a)

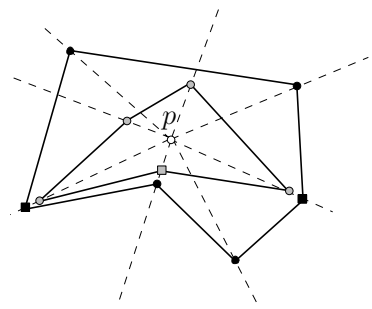

b)

Fig. 2. a) Polygons $P_{R}$ and $R_{B}$; and b) cycles $C_{R}$ and $C_{B}$.

If we consider white points instead Steiner ones, the following statement holds,

Theorem 3.2 Let $R \cup B$ a set of $n$ red and blue points. $2 n$ white points are always sufficient and sometimes necessary to obtain a 2-factor.

Proof. Regarding to the necessary condition it suffices to consider the points on a circle, being the white points on a hemisphere and the colored points alternated in the opposite one, as it is shown in Figure 3.

To prove that $2 n$ white points are always sufficient we make use of the Equitable Subdivision Theorem [4], that establishes that we can divide the plane in $n$ convex sets containing just a colored and two white points. The union of the triangles obtained gives rise to the 2 -factor. 


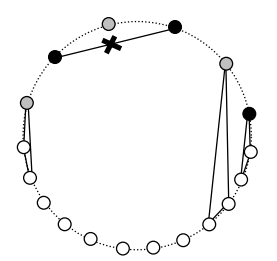

Fig. 3. To join two colored points left a third isolated, so the 2 -factor must be made including two white points.

Theorem 3.2 cannot be extended to 3 -factors, because points can be in convex position, as we saw in Figure 3, and any four points will give rise to an $K_{4}$ with self-intersections. The same happened when $k=4$ or 5 . But, what happened if we consider Steiner points? There is one obvious result

Theorem 3.3 Let $R \cup B$ a set of $n$ red and blue points. $O(n)$ Steiner points are always sufficient and sometimes necessary to obtain a $k$-factor, with $1 \leq$ $k \leq 5$.

Nevertheless the constants involved can be reduced with a more careful studying going down to $n$ Steiner points for 3 -factors, $5 n / 3$ for 4 -factors, and $26 / 3$ for 5 factors.

\section{Open problems}

The main problems that leave unsolved are related to the combine use of white and Steiner points to obtain $k$-factors. The only result in this sense concerns to 2-factors, and even in this case it would be interesting to study if it possible to reduce the number of Steiner points involved.

\section{References}

[1] A. Dumitrescu and R. Kaye. Matching colored points in the plane: Some new results. Computational Geometry, 19:69-85, 2001.

[2] A. Kaneko and M. Kano. Discrete geometry on red and blue points in the plane a survey-. Discrete and Computational Geometry. Algorithms and Combinatorics, 25:551-570, 2003.

[3] L. C. Larson. Problem-Solving Through Problems. Springer, New York, 1983.

[4] T. Sakai. Balanced convex partitions of measures in $\mathrm{R}^{2}$. Graphs and Combinatorics, 18(1):169-192, 2002. 